\title{
Nutzen und Sicherheit der Behandlung von Pleura- ergüssen mit dem PleurX-Katheter: eine retrospektive Analyse
}

\author{
Efficacy and Safety in the Treatment of Pleural Effusions with the \\ PleurX Catheter: A Retrospective Analysis
}

Autoren

Institut
M. Blaukovitsch, A. Strassburg, E. Müller, P. Zabel, H.-P. Hauber

Medizinische Klinik, Forschungszentrum Borstel, Borstel eingereicht 6.2 .2011

akzeptiert nach Revision 16.3.2011

\section{Bibliografie}

DOI http://dx.doi.org/ 10.1055/s-0030-1256364

Online-Publikation: 21. 4. 2011

Pneumologie 2011; 65 :

558-564 ๔ Georg Thieme

Verlag KG Stuttgart · New York ISSN 0934-8387

Korrespondenzadresse PD Dr. Hans-Peter Hauber Medizinische Klinik

Forschungszentrum Borstel Parkallee 35

23845 Borstel

hphauber@fz-borstel.de

\section{Zusammenfassung \\ $\nabla$}

Hintergrund: Bei Patienten mit rezidivierenden Pleuraergüssen unterschiedlicher Genese sind die Behandlungsergebnisse teilweise enttäuschend. Ziel dieser retrospektiven Beobachtungsstudie war es, den Nutzen und die Sicherheit eines subkutan getunnelten Pleurakatheters (PleurX-Katheter, Denver Biomedical, USA) zu untersuchen. Die chirurgische pleurale Dekortikation und die Talkum-Pleurodese galten bislang als effektive Behandlungsmöglichkeiten von rezidivierenden, in erste Linie malignen Pleuraergüssen. Der PleurX-Katheter bietet eine alternative Therapieoption vor allem bei eingeschränkter Lebenserwartung.

Methoden und Ergebnisse: 21 Patienten wurden retrospektiv untersucht. Bei 11 Patienten wurde sofort der PleurX-Katheter eingesetzt, die restlichen 10 Patienten wurden initial mit einer Pleurodese versorgt und bekamen bei nachlaufendem Erguss den PleurX in einem zweiten Schritt implantiert. Die mittlere Überlebenszeit nach Erstdiagnose der Grunderkrankung betrug 25 Wochen. Im Durchschnitt konnten $725 \mathrm{ml}$ Ergussmenge pro Woche abgelassen werden, wobei der Katheter bei insgesamt 16 Patienten bis zum Tod 1-2-mal in der Woche benutzt wurde. Die Komplikationsrate lag im Vergleich zu anderen Therapieoptionen im akzeptablen Bereich (19\%). Ein direkter Überlebensvorteil oder eine Verbesserung des klinischen Ergebnisses der einzelnen Therapieoptionen (PleurX alleine vs. Pleurodese/ PleurX im Anschluss) konnte statistisch nicht gezeigt werden. Interessanterweise war die geförderte Menge an Pleuraerguss pro Woche indirekt mit der Überlebenszeit nach PleurX-Implantation korreliert.

\section{Abstract \\ V}

Background: The purpose of this retrospective study was to investigate the efficacy and safety of an indwelling pleural device (PleurX, Denver Biomedical, USA) for the treatment of recurrent pleural effusions. In cases when life expectancy tends to be very short and also surgical decortication is not recommended, pleurodesis is another treatment option but requires complete drainage of the whole pleural fluid for optimal results which is sometimes hard to achieve. The PleurX catheter is an alternative therapeutic option.

Methods and Results: We retrospectively analysed the clinical data from a total of 21 patients who were treated with a PleurX alone (11 patients) or who initially received pleurodesis and afterwards a PleurX catheter (10 patients). Mean survival was 25 weeks after initial diagnosis of the underlying disease. The mean amount of pleural effusion drained per week was $725 \mathrm{~mL}$. 16 patients used the catheter until they died at least 1-2 times a week. The complication rate was $19 \%$ and thus within a reasonable range when compared to other treatment options for recurrent pleural effusions. There was no statistically significant difference in clinical outcome in both groups (pleurodesis and subsequent PleurX vs. PleurX alone). The amount of evacuated pleural effusion was inversely correlated with the remaining life time.

Conclusion: The use of an indwelling pleural device is a safe alternative treatment option for patients with chronic pleural effusions and trapped lung signs. We should be aware of this device and propagate its use at an earlier stage of malignant diseases with recurrent pleural effusions, especially when the remaining life time is short. 
Schlussfolgerung: Unsere Daten zeigen, dass durch die Anlage eines PleurX-Katheters eine sichere und effektive Behandlung rezidivierender Pleuraergüsse möglich ist. Bei einer fachgerechten Anwendung ist eine gute Funktionalität des PleurX gewährleistet. Bei schlechter Lungenentfaltung im Rahmen der Versorgung von malignen, rezidivierenden Pleuraergüssen, vor allem bei Patienten mit einer deutlich eingeschränkten Lebenserwartung, sollte die Möglichkeit der frühen Implantation eines PleurX-Katheters erwogen werden.

\section{Einführung}

Maligne Pleuraergüsse stellen eine häufige Komplikation fortgeschrittener Tumorerkrankungen unterschiedlichster Ätiologie in einer palliativen Situation dar. Kommt es - meist im Rahmen einer malignen Erkrankung oder einer Entzündung - zu einer Störung der Kapillarpermeabilität, bildet sich ein Exsudat. Auch eine Lymphabflussstörung der Pleura parietalis kann hierzu führen. Erkrankungen mit einer Erhöhung des hydrostatischen oder einer Verminderung des onkotischen Drucks führen zu einer vermehrten Produktion von pleuralem Transsudat. Die häufigsten Beispiele hierfür sind die Linksherzinsuffizienz mit vermehrtem Druck im kleinen Kreislauf, beziehungsweise das nephrotische Syndrom oder die Leberzirrhose mit einem manifesten Eiweißmangel und einem dadurch bedingten verminderten onkotischen Druck. Die Differenzierung zwischen Transsudat und Exsudat erfolgt anhand verschiedener laborchemischer Kriterien des allfälligen Punktats (Light Kriterien: Eiweißgehalt, spezif. Gewicht, LDH, Cholesterin, pH, Glucosegehalt).

$\mathrm{Zu}$ diagnostischen Schwierigkeiten kann es hierbei aufgrund der veränderten klinischen Chemie bei Transsudaten kommen, welche bereits länger diuretisch behandelt sind (Vortäuschung eines Exsudates). Andere, insgesamt eher seltene Gründe für Pleuraergüsse sind Läsionen des Ductus thoracicus (Chylothorax), Traumata (Hämatothorax, Chylothorax) oder Syndrome (z. B. MeiggsSyndrom, Yellow-Nail-Syndrom). Auch im Falle größerer Lungenatelektasen kann es durch die Verschiebung der Druckgradienten zur Transsudatbildung kommen. Wichtig ist diese Tatsache vor allem deswegen, weil so auch bei einem zentral obstruierenden Bronchuskarzinom Pleuraergüsse entstehen können, welche jedoch aufgrund der Tumorfreiheit der Pleura nicht als maligne zu werten sind (paramaligner Erguss) und somit keine Kontraindikation für einen kurativen Therapieansatz (Operation, Bestrahlung) bedeuten.

Ein persistierender Pleuraerguss (in der Regel exsudative intrapleurale Ergüsse), zusammen mit einer nicht expansionsfähigen Lunge, ist ein schwieriges klinisches Problem mit wenigen Behandlungsoptionen $[1,2,18]$.

Die Anlage einer Thoraxsaugdrainage (TSD) mit einer anschließenden Talkumpleurodese ist das am häufigsten durchgeführte medizinische Behandlungsverfahren. Nach Anlage des Drainagesystems ist aber die Bildung von gekammerten Kompartimenten innerhalb des Pleuraergusses ein limitierender Faktor für eine erfolgreiche Verklebung der Pleurablätter durch eine Pleurodese. Eine entsprechende Reexpansion der Lunge ist dann wiederum nicht möglich und erschwert das weitere therapeutische Vorgehen bei diesen Patienten [3]. Komplizierte und für den Patienten belastende chirurgische Verfahren wie die Pleuradekortikation sind weiterhin nur in Ausnahmefällen gerechtfertigt, da sie oft schwere postinterventionelle Komplikationen nach sich ziehen [4]. Wiederkehrende Pleurapunktionen, wenngleich dies ein ein- faches Verfahren zur Symptomlinderung von Pleurergüssen darstellt, verlangen vom Patienten regelmäßige ambulante Vorstellungen oder kurze stationäre Aufenthalte. Dies geht auf der einen Seite auf Kosten wertvoller verbleibender Lebenszeit des Patienten, auf der anderen Seite belastet es die Gesundheitssysteme mit überproportionalen finanziellen Ausgaben. Eine weitere alternative, wenn auch etwas überholte Methode, stellen pleuroperitoneale Shuntsysteme dar. Sie verlangen aber eine hohe Patientencompliance und bilden häufig Metastasen in der Peritonealhöhle aus [5].

Die oben erwähnte Pleurodese stellt bei nicht gekammerten Ergüssen sicherlich weiterhin den Goldstandard dar, wenngleich der Erfolg dieser Methode primär von der Durchführung abhängig ist (Talkumpleurodese über liegende Thoraxsaugdrainage oder mit Hilfe einer Poudrage unter thorakoskopischer Sicht). Hier ist die thorakoskopische Poudrage der Talkumpleurodese über TSD aufgrund besserer Verteilung des Talkumpuders unter Sicht vorzuziehen. Eine vollständige Entfaltung der Lunge und weitgehends „trockene“ intrapleurale Verhältnisse sind jedoch Voraussetzung für eine erfolgreiche Verklebung der Pleurablätter mittels Talkumpleurodese, -poudrage.

Da Patienten mit einem rezidivierenden/malignen Pleuraerguss per se eine schlechte Überlebensprognose haben, muss die durchgeführte Behandlung der symptomatischen Pleuraergüsse eine angemessene kurze Rekonvaleszenzphase mit sich bringen. Unter diesen Umständen stellt die Implantation eines getunnelten, intrapleuralen Katheters (PleurX, Denver Biomedical, USA) eine alternative Behandlungsmöglichkeit dar.

Neue Untersuchungsdaten von Efthymiou et al. zeigten, dass eine Verbesserung der Dyspnoe und Abnahme der Schmerzen sowie Steigerung der körperlichen Belastbarkeit des Patienten Hauptargumente für eine PleurX-Implantation darstellten [5]. Murthy et al. fanden heraus, dass ein PleurX-Katheter für die Behandlung von chronischen Pleuraergüssen außergewöhnlich gut von den Patienten vertragen wurde. Das komplikationsfreie Zeitintervall für nicht-gekammerte Pleuraergüsse konnte mit den Patienten, welche eine Talkumpleurodese erhielten, verglichen werden. Dies ist ein weiterer wichtiger Punkt neben der niedrigen Komplikationsrate sowie der leichten Handhabung des PleurX-Katheters mit hohem Patientenkomfort, diese Methode als palliativen Bestandteil eines multimodalen Behandlungskonzeptes anzusehen [6-9].

Der PleurX-Katheter wird in unserer Klinik seit drei Jahren systematisch eingesetzt. Das Ziel dieser retrospektiven Studie war es, den Nutzen und die Sicherheit des PleurX-Katheters zu untersuchen. Hierzu wurden die klinischen Daten von Patienten, bei denen eine Implantation eines PleurX-Katheters zur Behandlung von chronischen Pleuraergüssen durchgeführt wurde, retrospektiv analysiert.

\section{Patienten und Methoden}

\section{$\nabla$}

\section{Patienten}

Klinische Daten von insgesamt 21 Patienten, die einen PleurXKatheter zwischen Januar 2007 und Januar 2010 erhalten haben, wurden in dieser retrospektiven Beobachtungsstudie eingeschlossen. Für die Auswertung der klinischen Daten wurde die Krankengeschichte, Telefoninterviews mit den Patienten selbst, ihren Verwandten oder ihren Hausärzten durchgeführt. Demografische Daten der Patienten, Zeitpunkt der Erstdiagnose der Grunderkrankung, Datum der Pleurodese (-Versuch) über TSD 


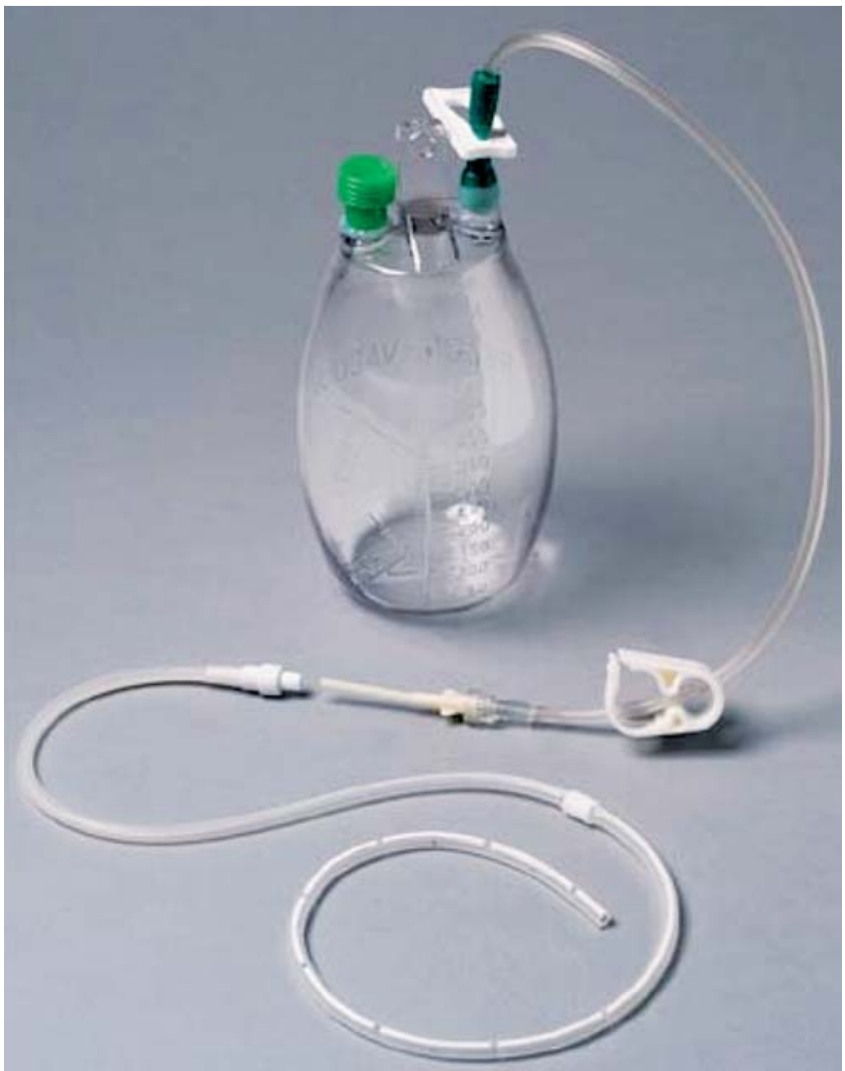

Abb. 1 PleurX ${ }^{\ominus}$-Katheter-System, das in unserer Klinik verwendet wird (Cardinal Health, Denver Biomedical, www.denverbio.com/physician_ pleurx_catheter.html).

und/oder Zeitpunkt der PleurX-Katheter-Implantation wurden gesammelt. Weiterhin wurde die Gesamtüberlebenszeit nach der initialen Diagnosestellung, nach PleurX-Katheter-Implantation und/oder Durchführung einer Pleurodese über TSD sowie Funktionalität des PleurX-Katheters evaluiert.

\section{Behandlungsoptionen}

Die Behandlung von Pleuraergüssen bei den Patienten wurde auf zwei verschiedene Arten durchgeführt. 10 Patienten erhielten am Anfang eine Thorakoskopie mit Anlage einer TSD und einen anschließenden Versuch einer Talkumpleurodese, gefolgt von einer PleurX-Katheter-Anlage wegen Misserfolgs der Pleurodese und nachlaufendem Erguss. Weitere 11 Patienten wurden sofort mit einem PleurX-Katheter bei persistierendem, sonografisch nachgewiesenem Sero-/Pneumothorax, fehlender Lungenexpansion sowie eingeschränktem Allgemeinzustand therapiert. Diese Patienten waren auch aufgrund des reduzierten Allgemeinzustandes und der fehlenden klinischen Durchführbarkeit einer Thorakoskopie mit Anlage eines iatrogenen Pneumothorax nicht für eine weiterführende Pleurodese über eine liegende Thoraxsaugdrainage geeignet ( $\bullet$ Abb. 1 u. 2).

Die Implantation des intrakutan getunnelten PleurX-Katheters wurde unter lokaler Anästhesie, aseptischen Bedingungen und unter leichter intravenöser Sedoanalgesierung an unserer Klinik nach internationalen Standards durchgeführt $[3,4,10]$. Zytologische und serologische Untersuchungen des Pleuraergusses mittels diagnostischer Punktion wurden vor Implantation des PleurX-Katheters oder der Thorakoskopie mit Anlage einer Thoraxsaugdrainage veranlasst. Die Patienten wurden anschließend von geschultem Trainingspersonal im Management des PleurX-

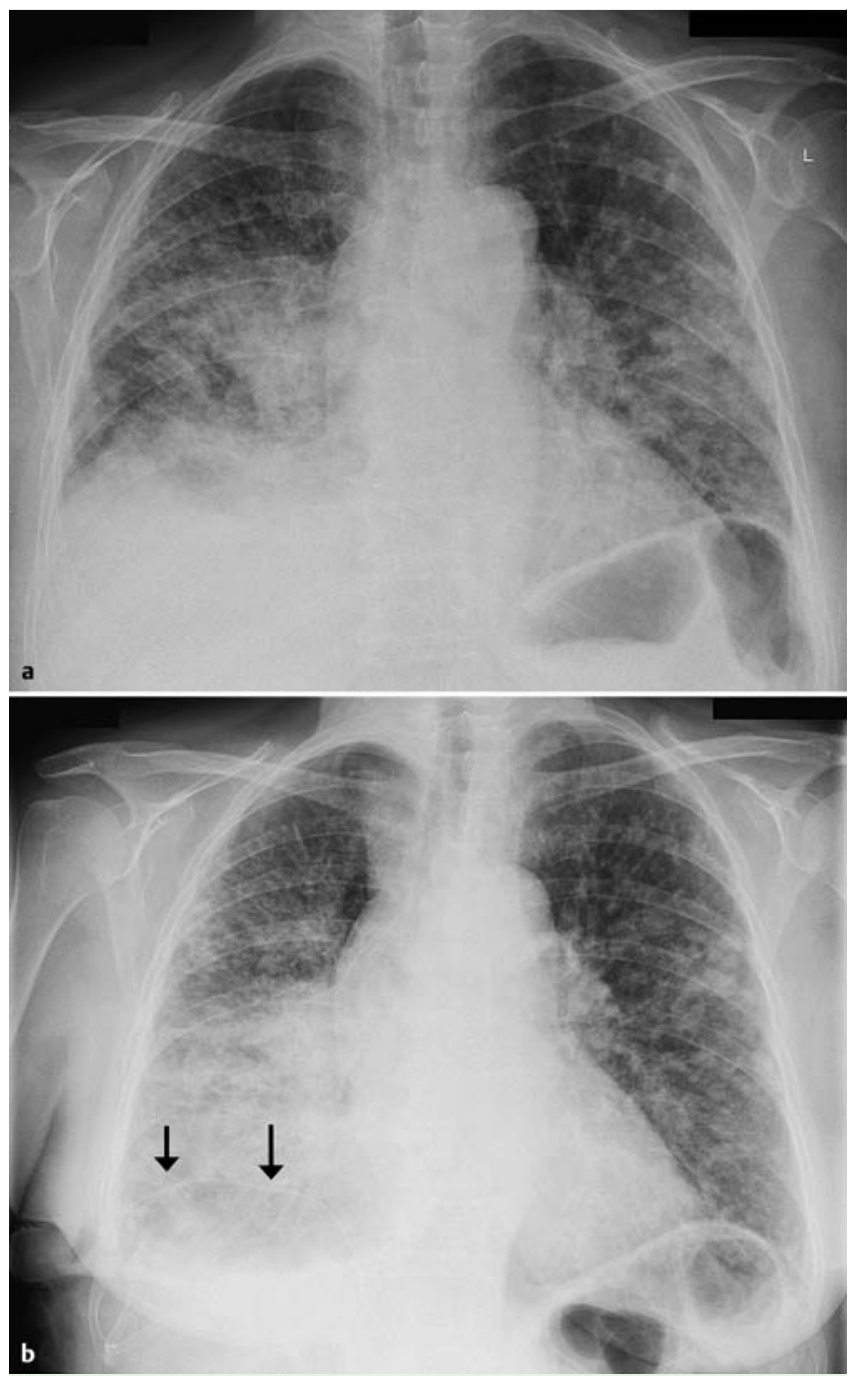

Abb. 2 Röntgenbild des Thorax von einem Patienten mit einem Pleuraerguss vor (a) und nach Anlage eines PleurX-Katheters rechts (b). Die zwei Pfeile zeigen die Lage des PleurX-Katheters.

Katheters durch Videopräsentationen und Informationsbroschüren instruiert. Zusätzlich wurden bei häuslicher Selbstversorgung die Hauskrankenpflege sowie die Angehörigen in der Handhabung des PleurX-Katheters unterwiesen.

\section{Statistische Analyse}

Die gewonnen Daten wurden für die Normalverteilung mit dem Test von Kolmogorov-Smirnov analysiert. Für die statistische Korrelation wurde der Korrelationskoeffizient nach Pearson verwendet. Eine Kaplan-Meier Überlebensanalyse wurde durchgeführt, um die zwei verschiedenen Behandlungsoptionen (Pleurodese und PleurX-Katheter im Anschluss versus PleurX-Katheter allein) miteinander zu vergleichen. Alle Werte sind als Mittelwert \pm Standardabweichung dargestellt, sofern nicht anders angegeben. 
Tab. 1 Demografische Daten der Patienten.

\begin{tabular}{|c|c|c|c|c|c|c|c|}
\hline Geschlecht & Alter & $\begin{array}{l}\text { Tumorart } \\
\text { oder Grund- } \\
\text { erkrankung }\end{array}$ & $\begin{array}{l}\text { Zeit zwischen Diagnose } \\
\text { „Pleuraerguss“ und Pleuro- } \\
\text { deseversuch mit Thorako- } \\
\text { skopie und Thorax- } \\
\text { schlaucheinlage in Tagen }\end{array}$ & $\begin{array}{l}\text { Zeit zwischen Diag- } \\
\text { nose „Pleuraerguss“ } \\
\text { und Anlage des } \\
\text { PleurX-Katethers in } \\
\text { Tagen }\end{array}$ & $\begin{array}{l}\text { Überlebens- } \\
\text { zeit nach } \\
\text { Anlage des } \\
\text { PleurX-Kathe- } \\
\text { ters in Tagen }\end{array}$ & $\begin{array}{l}\text { Gesamtüber- } \\
\text { leben nach } \\
\text { initialer } \\
\text { Diagnose in } \\
\text { Wochen }\end{array}$ & $\begin{array}{l}\text { Menge } \\
\text { in } \mathrm{ml} P E \\
\text { pro Wo. }\end{array}$ \\
\hline $\mathrm{m}$ & 60 & $\mathrm{NSCLC}$ & keine Thorakoskopie & 33 & 60 & 12 & 800 \\
\hline $\mathrm{m}$ & 74 & PM & keine Thorakoskopie & 20 & 35 & 11 & n. n. \\
\hline $\mathrm{m}$ & 83 & PM & 2 & 20 & 209 & 35 & n. n. \\
\hline $\mathrm{m}$ & 74 & NSCLC & 2 & 27 & 206 & 28 & 200 \\
\hline $\mathrm{m}$ & 65 & PM & 34 & 56 & 242 & 40 & 500 \\
\hline w & 85 & PM & 2 & 62 & 90 & 20 & 700 \\
\hline w & 64 & $\mathrm{NSCLC}$ & 14 & 43 & 55 & 13 & 500 \\
\hline $\mathrm{m}$ & 83 & $\begin{array}{l}\text { Linksherzversa- } \\
\text { gen [NYHA IV] }\end{array}$ & keine Thorakoskopie & 0 & 3 & 0 & 600 \\
\hline $\mathrm{m}$ & 63 & NSCLC & 194 & 316 & 4 & 50 & n. n. \\
\hline $\mathrm{m}$ & 67 & Nieren-Tumor & keine Thorakoskopie & 17 & 30 & 31 & 1200 \\
\hline $\mathrm{m}$ & 64 & SCLC & keine Thorakoskopie & 0 & 33 & 8 & 1200 \\
\hline w & 81 & NSCLC & keine Thorakoskopie & 3 & 37 & 2 & 750 \\
\hline w & 75 & PM & keine Thorakoskopie & 123 & 12 & 66 & n. n. \\
\hline $\mathrm{m}$ & 48 & $\begin{array}{l}\text { Hodgkin } \\
\text { Lymphom }\end{array}$ & keine Thorakoskopie & 35 & 4 & 57 & n. n. \\
\hline $\mathrm{m}$ & 73 & SCLC & keine Thorakoskopie & 14 & 49 & 9 & 1400 \\
\hline w & 72 & PM & keine Thorakoskopie & 11 & 165 & 24 & 700 \\
\hline $\mathrm{m}$ & 49 & NSCLC & 26 & 54 & 10 & 25 & 1400 \\
\hline $\mathrm{m}$ & 72 & PM & 6 & 12 & 112 & 24 & 300 \\
\hline w & 43 & $\mathrm{NSCLC}$ & 2 & 9 & 47 & 84 & n. n. \\
\hline w & 40 & Brust-Tumor & keine Thorakoskopie & 8 & 8 & 157 & 2400 \\
\hline $\mathrm{m}$ & 52 & $\begin{array}{l}\text { Speicheldrüsen- } \\
\text { Tumor }\end{array}$ & 1 & 31 & 25 & 232 & n. n. \\
\hline
\end{tabular}

NSCLC: Nicht kleinzelliges Bronchialkarzinom; SCLC: Kleinzelliges Bronchialkarzinom; PM: Pleuromesotheliom; PE: Pleuraerguss; ml: Milliliter NYHA: New York Heart Association; n. n.: keine Angaben; m: männlich; w: weiblich

\section{Ergebnisse}

\section{Demografische Daten}

Die untersuchte Patientengruppe $(\mathrm{n}=21)$ schloss 14 männliche und 7 weibliche Personen ( $\mathrm{m}: \mathrm{w}$ Verhältnis $2: 1)$ ein. Das durchschnittliche Alter des Patientenkollektivs betrug $66 \pm 13$ Jahre. 16 Patienten litten an einer bösartigen, pulmonalen Grundkrankheit (7 NSCLC, 2 SCLC, 7 Pleuramesotheliome). Davon wiederum hatten 12 Patienten einen exsudativen Pleuraerguss nach den LightKriterien, 2 Patienten hatten definitionsgemäß einen paramalignen Erguss und bei 2 weiteren Patienten war eine laborchemische Untersuchung des Pleuraergusses aus technischen Gründen nicht möglich. 6 Patienten von diesen 16 hatten einen zytologisch positiven Nachweis des pulmonalen Primärtumors im Pleuraerguss, bei weiteren 6 war eine eindeutige zytologische Differenzierung aus dem Pleuraerguss nicht möglich, 2 Patienten hatten einen zytologisch negativen Befund. 4 Patienten litten unter einem extrapulmonalen Malignom (Nierenzell-Karzinom, Morbus Hodgkin kombiniert mit einem Endothelsarkom, Mamma-Karzinom und Karzinom der Gl. submandibularis) und ein Patient an einer fortgeschrittenen Linksherzinsuffizienz.

14 Patienten erhielten eine Chemotherapie und/oder eine chirurgische Behandlung vor der PleurX-Katheter-Implantation, wohingegen bei 7 Patienten eine palliative Behandlung aufgrund der fortgeschrittenen Grunderkrankung mit alleiniger PleurXKatheter-Anlage angestrebt wurde. $\bigcirc$ Tab. 1 fasst die demografischen Daten zusammen.

\section{Pleuraergüsse und Katheter-Komplikationen}

Bei 14 Patienten konnte im Durchschnitt 725 ml Pleuraflüssigkeit pro Woche über den PleurX-Katheter drainiert werden. Eine genaue Angabe der drainierten Flüssigkeitsmenge der verbleibenden Patienten konnte nicht gemacht werden. Der Katheter wurde von 16 Patienten bis kurz vor ihrem Tod verwendet. Katheterbezogene Komplikationen lagen bei 19\% (4 von 21). Zwei PleurXKathetersysteme mussten wegen Infektionen nach zwei Wochen explantiert werden. Zwei weitere Katheter wurden wegen Fehlfunktion nach drei Wochen entfernt. Ein Katheter wurde bei einer chirurgischen Pleurektomie nach 12 Wochen entfernt (४ Tab. 2).

Tab. 2 Komplikationen nach PleurX-Katheter (4 von 21 Patienten).

$\begin{array}{ll}\text { Ursache } & \text { Anzahl } \\ \text { Infektion } & 2 \\ \text { Fehlfunktion } & 2\end{array}$

\section{Überleben}

Die mittlere Überlebenszeit aller Patienten nach Erstdiagnose der Grunderkrankung lag bei 25 Wochen. Im Vergleich dazu betrug die mittlere Überlebenszeit 20 Wochen nach der Diagnosestellung bei Patienten, die vor PleurX-Katheter-Implantation einen Pleurodeseversuch durch eine TSD erhielten. Demgegenüber steht eine mittlere Überlebenszeit von 8 Wochen bei den Patienten, die sofort mit einem PleurX-Kathetersystem versorgt wurden ( $\bullet$ Abb. 3 u. 4). 


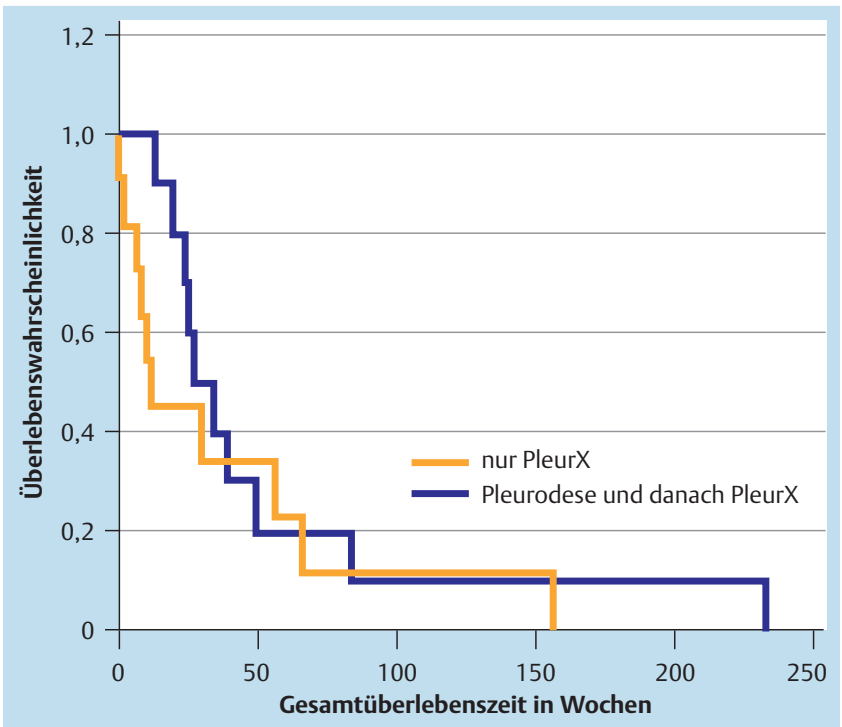

Abb. 3 Gesamtüberlebenszeit beider Patientengruppen nach Erstdiagnose der Grunderkrankung mit und ohne vorheriger Pleurodese. Kaplan-Meier-Kurve.

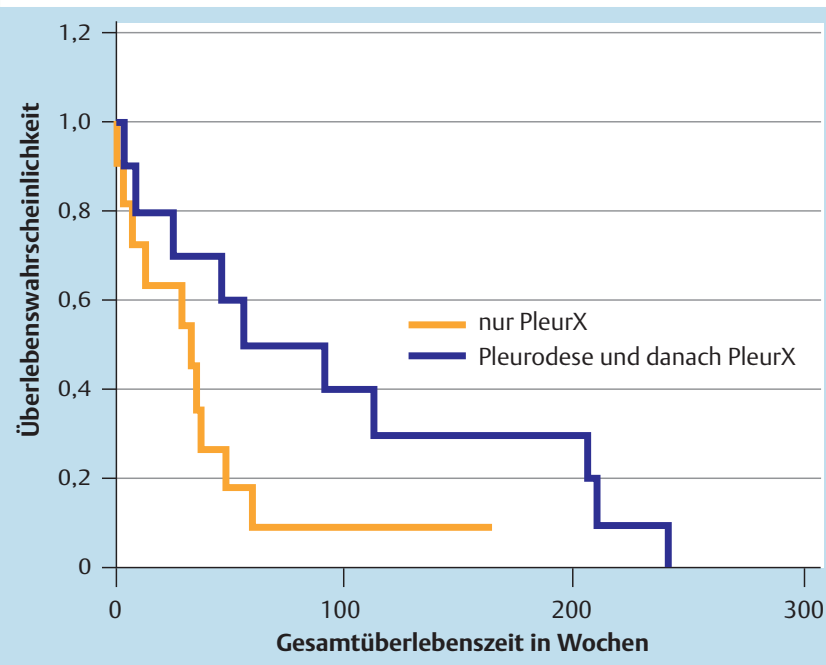

Abb.4 Gesamtüberlebenszeit beider Patientengruppen nach sofortiger PleurX-Katheter-Implantation oder nach vorheriger Pleurodese mit anschließender PleurX-Katheter-Implantation. Kaplan-Meier-Kurve.

Die mittlere Zeitspanne zwischen dem Pleurodese-Versuch und der Anlage des PleurX-Katheters betrug 28 Tage. Es gab keinen statistisch signifikanten Überlebensvorteil zwischen den zwei Behandlungsoptionen (PleurX-Katheter nach Pleurodeseversuch versus unmittelbare PleurX-Katheter-Implantation nach Diagnosestellung; $\mathrm{p}>0,05)$.

Interessanterweise war die drainierte Pleuraergussmenge pro Woche invers proportional zur verbleibenden Lebenszeit nach PleurX-Anlage $(\mathrm{p}<0,01$; Korrelations-Koeffizient von Pearson: $-0,6)$.

\section{Lebensqualität}

In der retrospektiven Befragung berichtete keiner der Angehörigen der Patienten von einer Einschränkung im alltäglichen Leben des Patienten durch den PleurX-Katheter. In 90\% der Fälle hatte der Patient eine bemerkenswerte Abnahme von pleuraerguss- bedingten Symptomen wie Dyspnoe oder thorakalen Schmerzen (๑ Abb.4).

\section{Diskussion}

Wir untersuchten in dieser retrospektiven Analyse das klinische Ergebnis eines intrakutan getunnelten Pleurakatheters als eine alternative Behandlungsoption für Patienten mit malignen, chronisch-rezidivierenden Pleuraergüssen, welche von einer Pleurodese aufgrund fehlender Lungenreexpansion nicht profitierten oder bei denen eine Talkumpleurodese beziehungsweise eine thorakoskopische Poudrage aufgrund des schlechten Allgemeinzustandes nicht möglich war.

Generell finden chirurgische Behandlungsoptionen von symptomatischen chronischen Pleuraergüssen bei Patienten, die an einer bösartigen Krankheit leiden, nur eingeschränkt Anwendung. Eine partielle Pleurektomie sollte nicht an Patienten mit einer erwarteten Überlebenszeit von weniger als 6 Monaten durchgeführt werden $[3,11]$. Wir gehen davon aus, dass alle unsere Patienten einen Vorteil durch die PleurX-Katheter-Anlage hatten. Insbesondere das Vermeiden einer Narkose und Anwendung einer milden Analgosedierung ist ein nennenswerter Vorteil bei der PleurX-Katheter-Anlage. Mehrere Studien mit einer 10-jährigen Durchführungsdauer bei pleuroperitonealen Shuntsystemen sind veröffentlicht worden $[12,14]$. Die Ergebnisse sind alle nicht sehr vielversprechend. Es sind vor allem technische Schwierigkeiten, die gegen diese Systeme sprechen. Die Pumpengröße ist für gewöhnlich sehr klein $\left(1,5 \mathrm{~cm}^{3}\right)$, und es sind oft mehrere Pumpensysteme notwendig, um eine ausreichende Menge an Pleuraflüssigkeit in die Peritonealhöhle zu drainieren. Dies führt oft zu iatrogenen, peritonealen Entzündungen mit nachfolgender Non-Compliance der Patienten. Nicht zu vernachlässigen ist die Tatsache, dass Tumorzellen in die Bauchhöhle ausgeschwemmt werden und so peritoneale Fernmetastasen bilden können, was wiederum in weiterer Folge zu Aszitesbildung führen kann $[6,9,13]$.

Die Thorakoskopie ist der Goldstandard, um Pleuraergüsse unklarer Ätiologie zu untersuchen. Es wird empfohlen, dass sowohl für die Diagnose als auch für Behandlung rezidivierender Pleuraergüsse eine Thorakoskopie durchgeführt werden sollte [6].

In der vorliegenden retrospektiven Studie sind jedoch nicht alle Pleuraergüsse thorakoskopisch abgeklärt worden. 9 Patienten erhielten eine Diagnose mit einer einfachen sonografisch gestützten Pleurapunktion, da eine Thorakoskopie mit einer iatrogenen Pneumothoraxanlage wegen des schlechten Allgemeinzustands der Patienten nicht mehr durchgeführt werden konnte. Es ist interessant, dass Patienten mit einem Pleuramesotheliom (PM) eine doppelt so hohe mittlere Überlebensrate nach PleurX-Implantation hatten wie Patienten mit einem primären Lungentumor. Es konnte zwar kein signifikanter statistischer Unterschied herausgearbeitet werden, aber immerhin lag der statische Medianwert bei den NSCLC/ SCLC- Patienten bei 47 Tagen und bei Patienten mit einem PM bei 112 Tagen. Der Grund hierfür ist rein spekulativ, aber nachdem PM bereits in einer sehr frühen Phase des Krankheitsverlaufs Pleuraergüsse ausbilden, führt dies wiederum zu einer frühen Symptomausbildung wie Dyspnoe und Schmerzen und in weiterer Folge zu einer früheren Anlage des PleurX-Katheters. Bei solchen Patienten mit einem PM sind oft Lungenanteile nicht mehr vollständig entfaltbar und diese profitieren von einer Pleurodese daher nur in wenigen Fällen. Diesen Patienten kann daher meist durch eine PleurX-Anlage bei weitem am besten geholfen werden. 
4 Patienten mit einer nicht pulmonalen, malignen Grunderkrankung starben innerhalb 17 Tage, nachdem der Pleuraerguss durch das PleurX-Katheter-System drainiert wurde. Weitere 4 Patienten starben bereits 8 Tage nach Anlage des PleurX-Systems. Hier muss man retrospektiv sicherlich kritisch mit der Indikationsstellung zur Anlage eines PleurX-Katheters umgehen. Bei diesen Patienten handelt es sich klinisch um eine sehr fortgeschrittene maligne Grunderkrankung beziehungsweise den Fall einer fortgeschrittenen Linksherzinsuffizienz. Es bleibt eine ärztliche Ermessenssache, ob man Patienten mit „End-stage“-Erkrankungen eine Symptomenlinderung durch regelmäßiges Ablassen des Ergusses mithilfe eines PleurX-Katheters auch nur für kurze Zeit anbieten möchte. Sicherlich zielt die Implantation eines PleurX-Katheters auf eine längere ambulante Nutzung ab, jedoch ist die schnelle und relativ einfache Anlage des Systems auch bei Patienten zu rechtfertigen, die eine sehr kurze zu erwartende Überlebenszeit haben und eine entsprechende Symptomlinderung dadurch bekommen.

Statistisch konnten wir zeigen, dass die drainierte wöchentliche Menge an Pleuraflüssigkeit invers mit der verbleibenden Überlebenszeit korreliert war, nachdem das PleurX-Katheter-System implantiert wurde. Dies könnte auf die Tatsache hinweisen, dass die absolute Menge an drainierter Pleuraflüssigkeit, z. B. pro Woche, als ein prädiktiver Parameter für die restliche Überlebenszeit verwendet werden kann. Wir können zwar keine absoluten Zahlen angeben, aber da die gesamte Überlebenszeit vom Tumorstadium respektive Tumormasse und der so erzeugten Menge an Pleuraflüssigkeit abhängt, könnte hier ein Zusammenhang bestehen. Limitierend ist anzumerken, dass dies nur bei 14 Patienten möglich war und wir daher eine generelle Aussage auf das ganze Patientenkollektiv nicht treffen können.

Der besondere Vorteil des PleurX-Katheter-Systems ist der getunnelte Teil in der Subkutis, was sich wiederum in einer akzeptablen Infektionsrate widerspiegelt. In der Literatur wird eine Komplikationsrate zwischen 3 und 15\% angegeben, was auch in unserer retrospektiven Analyse bestätigt wurde. Bei den 2 Patienten, welche den Katheter aufgrund einer Infektion entfernen lassen mussten, war im Vorfeld keine Talkumpleurodese durchgeführt worden. Eine vorausgegangene Chemotherapie kann als Risikofaktor (z.B. durch eine Neutropenie) ausgeschlossen werden, da kein direkter zeitlicher Zusammenhang erkennbar war. In unserem Patientenkollektiv lag die Infektionsrate bei 10\% (2 von 21 Patienten). Ob sich dieser Sachverhalt in einem größeren Patientenkollektiv auch darstellen lässt, kann aufgrund der insgesamt geringen Fallzahl nicht bestätigt werden.

In unserer Klinik sahen wir weiter keine Probleme während des Einheilungsprozesses des Schlauchsystems durch die minimalinvasive Anlagetechnik. Wie in vorherigen Studien empfohlen, setzten wir keine antibiotische Kurzzeitprophylaxe ein $[5,10]$. Alle unsere Patienten litten an einer nicht heilbaren Grunderkrankung im Endstadium. Die gesamte Komplikationsrate mit $19 \%$ (4 aus 21) war überschaubar und unterstreicht den Vorteil dieses intrapleuralen Katheters.

Aufgrund unserer Daten sind wir der Auffassung, dass der PleurX-Katheter für die Linderung von Pleuraerguss-spezifischen Symptomen in einer definierten Gruppe von Patienten früher eingesetzt werden kann $[16,17]$. Weder die Gruppe von Patienten, die zuerst eine Pleurodese (bzw. einen -Versuch) über eine TSD und im Anschluss danach einen PleurX-Katheter erhielten, noch die Patienten, die eine unmittelbare Implantation eines PleurX-Katheters bekamen, hatten eine statistisch unterschiedliche Überlebenszeit. Kritisch zu beurteilen ist auch hier die Frage der Patientenauswahl für eine Pleurodese über eine liegende TSD. Alle unsere Patienten, welche vor PleurX-Anlage eine Thorakoskopie und einen Pleurodeseversuch erhielten, zeigten keine Kontraindikationen (fehlende Lungenexpansion, gekammerter Erguss) für eine Talkumpleurodese. Vielmehr sind wir retrospektiv der Meinung, dass wahrscheinlich die Methode der Talkumpleurodese generell über eine liegende TSD als nicht effizient genug betrachtet werden kann. Ob hier eine thorakoskopische Poudrage von Vorteil gewesen wäre, bleibt rein spekulativ. Im Falle einer Pleurodese durch eine TSD muss ein etwa 7 Tage langer, kostenintensiver Krankenhausaufenthalt in Kauf genommen werden. Wir würden daher anhand der Studienlage und unserer Daten eine Pleurodese mittels TSD für Patienten, deren Lebenserwartung nur wenige Wochen beträgt, nicht mehr unbedingt empfehlen [9].

Die kleine Patientenanzahl und der retrospektive Charakter unserer Analyse sind Hauptdefizite. Außerdem konnten wir nur bei 14 Patienten rückblickend die mittlere Menge drainierter Pleuraflüssigkeit über den PleurX-Katheter bestimmen. Dennoch war es möglich zu zeigen, dass die Mehrheit unserer Patienten vom Gebrauch des Katheters anhand der Verbesserung der Symptome durch den abgelassenen Pleuraerguss und Besserung ihrer Lebensqualität vom frühzeitigen Einsatz des PleurX-Katheters profitierten. Andere chirurgische Behandlungsmethoden wie eine partielle Dekortikation der Pleura sollten keine alternative Behandlungsauswahl für diese Patienten in einem multimodalen Therapiekonzept sein.

Zusammenfassend denken wir, dass der PleurX-Katheter eine gute Therapieoption bei chronischen und rezidivierenden Pleuraergüssen darstellt, insbesondere wenn keine volle Entfaltung der Lunge nach Ergussdrainage zu erwarten oder die verbleibende Lebenszeit überschaubar ist.

\section{Interessenkonflikt}

$\nabla$

Die Autoren geben an, dass kein Interessenkonflikt besteht.

\section{Literatur}

1 Warren WH, Kim AW, Liptay MJ. Identification of clinical factors predicting PleurX catheter removal in patients treated for malignant pleural effusion. Eur J Cardiothorac Surg 2008; 33: 89-94

2 Warren $W$. Talc pleurodesis for malignant pleural effusions is preferred over the PleurX catheter [contrary position]. Ann Surg Oncol 2007; 10: $2700-2701$

3 Sioris T, Sihvo E, Salo J et al. Long-term indwelling pleural catheter [PleurX] for malignant pleural effusion unsuitable for talc pleurodesis. Eur J Surg Oncol 2009; 35: 546-551

4 Efthymiou CA, Masudi T, Thorpe JA et al. Malignant pleural effusion in the presence of trapped lung. Five-year experience of PleurX tunnelled catheters. Interact Cardiovasc Thorac Surg 2009; 9: 961 - 964

5 Putnam JB Jr., Walsh GL, Swisher SG et al. Outpatient management of malignant pleural effusion by a chronic indwelling pleural catheter. Ann Thorac Surg 2000; 69: 369-375

6 Murthy SC, Okereke I, Mason DP et al. A simple solution for complicated pleural effusions. J Thorac Oncol 2006; 1: 697-700

7 Pollak JS. Malignant pleural effusions: treatment with tunneled longterm drainage catheters. Curr Opin Pulm Med 2002; 8: 302 - 307

8 PollakJS, Burdge CM, Rosenblatt $M$ et al. Treatment of malignant pleural effusions with tunneled long-term drainage catheters. J Vasc Interv Radiol 2001; 12: $201-208$

9 Tremblay A, Michaud G. Single-center experience with 250 tunnelled pleural catheter insertions for malignant pleural effusion. Chest 2006; 129: $362-368$

10 Van den Toorn LM, Schaap E, Surmont VF et al. Management of recurrent malignant pleural effusions with a chronic indwelling pleural catheter. Lung Cancer 2005; 50: $123-127$ 
11 Spector M, Pollak JS. Management of malignant pleural effusions. Semin Respir Crit Care Med 2008; 29: 405-413

12 Petrou M, Kaplan D, Goldstraw P. Management of recurrent malignant pleural effusions. The complementary role talc pleurodesis and pleuroperitoneal shunting. Cancer 1995; 75: $801-805$

13 Courtney A, Nemcek AA Jr., Rosenberg S et al. Prospective evaluation of the PleurX catheter when used to treat recurrent ascites associated with malignancy. J Vasc Interv Radiol 2008; 19: 1723-1731

14 Rosenberg S, Courtney A, Nemcek AA Jr. et al. Comparison of percutaneous management techniques for recurrent malignant ascites. J Vasc Interv Radiol 2004; 15: 1129-1131
15 Herlihy JP, Loyalka P, Gnananandh $J$ et al. PleurX catheter for the management of refractory pleural effusions in congestive heart failure. Tex Heart Inst J 2009; 36: 38-43

16 Bertolaccini L, Zamprogna C, Barberis L et al. Malignant pleural effusions: review of treatment and our experience. Rev Recent Clin Trials 2007; $2: 21-25$

17 Stather DR, Tremblay A. Use of tunneled pleural catheters for outpatient treatment of malignant pleural effusions. Curr Opin Pulm Med 2007; 13: 328

18 Schindler O, Auer W. Pleuraerguss. INTERN 2009; 22: 5-9 